\section{Telemedicine and rural care}

$\mathrm{T}$ elemedicine can be a marvellous bridge between rural hospitals and tertiary centres, but the spectacular case recounted by Bruce Campana and colleagues ${ }^{1}$ may not illustrate this point well. Telemedicine works best when it does not degrade local care. The article seems to imply that the rural hospitals involved would deny patients proper care without the video presence of an urban specialist. This implication insults the staff of rural hospitals without access to telemedicine, who competently handle a variety of serious conditions with outcomes equal to or better than those achieved by their tertiary care counterparts.

Drilling a burr hole, although a rare procedure, is not a skill requiring advanced neurosurgical expertise. Rural doctors have sometimes had to do craniotomies themselves, the skill being swiftly acquired. ${ }^{2}$ Perhaps the local hospital described by Campana and colleagues lacked training and equipment, given that many rural facilities are becoming triage centres that also offer geriatric and palliative care. More likely, however, the telemedicine - while providing a measure of reassurance for what is probably a superb rural hospital - reinforced the authors' notion that burr holes, trauma or any advanced care cannot be handled competently without an urban specialist.

No one knows the financial cost of making telemedicine widely available in rural Canada, but enhancing local skills and equipment (through provision of CT scanners and operating rooms along with well-trained generalists) could probably be achieved at a fraction of that expense. The latter option would improve morale and outcomes more than images on a video screen. Telemedicine could then be used in a more selective, effective manner than the authors' "protean" hopes.

\section{Keith MacLellan}

Rural physician

Shawville, Que.

\section{References}

1. Campana BA, Jarvis-Selinger S, Ho K, Evans WL, Zwimpfer TJ. Use of telemedicine for an emergency craniotomy in a pediatric trauma. CMA7 2004;171(5):444-6.

2. MacLellan K. The occasional burr hole. Can $\mathcal{f}$ Rural Med 1998;3(4):223-5.

Competing interests: None declared.

DOI:10.1503/cmaj.1041469

\section{A life in the country}

Tames Rourke ${ }^{1}$ suggests numerous strategies for increasing the enrolment of students of rural origin, in the hope of increasing the supply of rural physicians. Yet these proposals fail to address the fact that many rural students will not return to small communities after training; furthermore, many nonrural students with the type of outdoor interests suggesting a likelihood to take up more isolated practices never leave the city where they trained. Even smaller cities are in a chronic human resource crisis.

One has only to look at the training of physicians to see why. To be accepted into medical school, candidates must already have completed a 4-year honours course, and they have often made 2 or 3 applications to med school before they are accepted. With a minimum of 6 years at medical school before they are free to decide where they will practise, these new doctors have already spent at least 10 years at school, often in the same large city. Given that many of them will have taken extra time in high school to ensure top grades for their initial university admission, the "young doctor" is, at 30 years of age, no longer young. Most will be married or in a commit- ted relationship, with the other partner established in his or her own career. Some will have children and mortgages. Their friends and social activities will be centred close to where they trained. Is it any wonder that they don't up and move even 50 miles from a major city?

The answer is not going to be a medical school in every community, because that would dilute the enormously important "centre of excellence" function of these institutions. Rather, the solution must include reducing the length of training. Making a 2-year premedical program the prerequisite for medical school, instead of a 4-year honours degree in an often unrelated subject, would go a long way to producing the kind of younger and more adventurous physicians we require.

\section{Paul Cary \\ Physician \\ Cambridge, Ont.}

\section{Reference}

1. Rourke J, for the Task Force of the Society of Rural Physicians of Canada. Strategies to increase the enrolment of students of rural origin in medical school: recommendations from the Society of Rural Physicians of Canada [editorial]. CMA7 2005;172(1):63-5.

DOI:10.1503/cmaj.1050019

$\mathrm{R}$ eaders concerned about the issue of increasing enrolment of rural students in medical school, as addressed by James Rourke, ${ }^{1}$ may be interested in a new initiative at Queen's University that is intended to better prepare students for the realities of rural life. "Professionals in Rural Practice: An Interdisciplinary Approach" is an elective course for undergraduate students in education and health sciences (medicine, nursing and rehabilitation therapy) and for graduate students in theology, which will be offered for the first time in fall 2005. Many issues of rural life are similar for people in all these professions. A research component will assess whether 
retention in rural areas is improved by this interdisciplinary elective. We hope that providing appropriate background and developing skills among students who are already interested in working in rural areas will lead them to stay longer in rural practice and give them greater satisfaction in their lives there.

\section{Denise Bowes}

Retired family physician

Athens, Ont.

Neil Hobbs

School of Medicine

Faculty of Health Sciences

Brian McAndrews

Faculty of Education

Jennifer Medves

School of Nursing

Faculty of Health Sciences

Margo Paterson

School of Rehabilitation Therapy

Faculty of Health Sciences

Elaine Smith

John H. Young

Queen's Theological College

Queen's University

Kingston, Ont.

\section{Reference}

1. Rourke J, for the Task Force of the Society of Rural Physicians of Canada. Strategies to increase the enrolment of students of rural origin in medical school: recommendations from the Society of Rural Physicians of Canada [editorial]. CMÄ̈ 2005;172(1):63-5.

Acknowledgement: The interdisciplinary course described in this letter is supported by a grant from the Bridge Street United Church Foundation.

DOI:10.1503/cmaj.1050031

$\mathrm{J}$ ames Rourke outlines many useful strategies to promote enrolment of students of rural origin in medical schools. ${ }^{1}$ Alex McPherson also raises several interesting issues related to the concept of rural training. ${ }^{2}$ Once students are in medical school, however, they need opportunities to learn in the geographic areas where we ultimately want them to practise.

Ontario now has 5 Distributed Medical Education (DME) programs, all funded by the Ministry of Health. Based in Thunder Bay, Sudbury, Collingwood, London and Perth, these programs have grown from their origins as coordinated opportunities for community-based medical school elec- tives, and they now place both core and elective learners in high-quality sites with faculty-appointed preceptors.

The Collingwood-based program, the Rural Ontario Medical Program (www.romponline.com), was established in 1988 and operates in partnership with the 5 (soon to be 6) Ontario medical schools. ROMP offers community-based rotations ranging from several weeks to well over a year in duration. Educational rotations are offered starting in the first year of medical school and continuing into clinical clerkship and residency; short-term and long-term rotations in family medicine and specialist programs are also offered. During their rotations in the communities, trainees may visit high schools to encourage rural students to consider a medical career. At the end of their formal education, we offer new physicians placement opportunities through our Community Development Office (www.cdoprogram.com). Once new graduates have been placed, we work with them to identify opportunities for them to become teachers for the program.

The DME programs have been successful in providing one solution to the rural physician shortage. A 10-year retrospective analysis in 2001 showed that $98(47 \%)$ of the 209 participants in ROMP are now practising in rural or underserviced communities. The 2 northern programs (based in Thunder Bay and Sudbury) have similar success rates.

As noted by the WONCA Working Party on Training for Rural Practice, ${ }^{3}$ "After a rural background, the next strongest factor associated with entering rural practice is undergraduate and postgraduate clinical experience in a rural setting." The opportunities offered by Ontario's DME programs will play a vital role in the future sustainability of community medical practice.

\section{Peter Wells}

Founding Executive Director

Rural Ontario Medical Program

Collingwood, Ont.

\section{References}

1. Rourke J, for the Task Force of the Society of Rural Physicians of Canada. Strategies to in- crease the enrolment of students of rural origin in medical school: recommendations from the Society of Rural Physicians of Canada [editorial]. CMÄ̈ 2005;172(1):63-5.

2. McPherson A. Sustainability of family medicine [letter]. CMA7 2005;172(2):157.

3. WONCA Working Party on Training for Rural Practice. Training for rural general practice. [place unknown]: World Organisation of Family Doctors; 1995. Available: www.globalfamilydoctor .com/aboutWonca/working_groups/rural_training /training/WONCAP.htm (accessed 2005 Mar 17).

DOI:10.1503/cmaj.1050082

\section{Perspectives on drug withdrawals}

D hysicians are the ultimate decision1 makers in the use of prescription drugs, but we depend on the integrity of pharmaceutical companies. We spend much of our time modifying risk factors for vascular disease, but in the past few years, 2 major classes of drugs have proven to be contributors to vascular disease. The first to fall were the menopausal replacement hormones, and now we are dealing with the fallout related to COX-2 inhibitors. ${ }^{1}$

When the COX-2 drugs arrived on the scene, I actually stopped prescribing older nonsteroidal anti-inflammatory drugs, because of the risk of upper gastrointestinal bleeding. I began to have doubts about the COX-2 inhibitors when some patients experienced gastric or intestinal problems anyway, and others seemed to experience rather severe hypertension.

The drug representatives showered me with so many samples that I seldom had to write a prescription. One rep gave me an entire case, which lasted for months. I tended to favour whichever COX-2 inhibitor was in my sample cupboard, but they are a hard sell now that 2 of them have been banned. $\mathrm{Pa}$ tients read newspapers too, but surely we physicians deserve to get bad news about drugs from the manufacturers well before it hits the papers.

My fear now is that those of my patients who received COX-2 inhibitors and who now have vascular disease may question my treatment, just as my menopausal patients have questioned replacement hormone therapy. Patients are surprisingly forgiving, but there is un- 